Supplement of Geosci. Model Dev., 10, 4393-4403, 2017

https://doi.org/10.5194/gmd-10-4393-2017-supplement

(C) Author(s) 2017. This work is distributed under

the Creative Commons Attribution 3.0 License.

(c) (1)

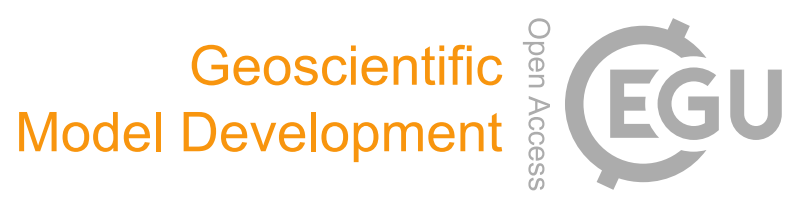

Supplement of

\title{
A JavaScript API for the Ice Sheet System Model (ISSM) 4.11: towards an online interactive model for the cryosphere community
}

Eric Larour et al.

Correspondence to: Eric Larour (eric.larour@jpl.nasa.gov)

The copyright of individual parts of the supplement might differ from the CC BY 3.0 License. 


\section{Supplement}

JavaScript compilation using ISSM is only supported on a subset of platforms. The port has been achieved on MacOSX platforms using the Emscripten suite of compilers, available at http://kripken.github.io/ emscripten-site. Here, we present the specific set of instructions regarding installation of ISSM with JavaScript

5 support. They build on the instructions for installation of the regular ISSM package, which can be found at http://issm.jpl.nasa.gov/download/

The main difference when dealing with compiling ISSM with JavaScript support turned on lies in the specific configuration that uses the Emscripten compiler. This configuration can be turned on by using the following configure arguments during the ISSM configuration phase:

\#/bin/bash

export $\mathrm{CC}=\mathrm{emcc}$

export $\mathrm{CXX}=\mathrm{em}++$

. /configure --prefix="\$ISSM_DIR/build-js" ।

--srcdir="\$ISSM_DIR" ।

--with-javascript ।

--disable-shared ।

--with-triangle-dir="\$HOME/externalpackages/triangle/install-javascript" ।

--with-gsl-dir="\$HOME/externalpackages/gsl/install-javascript" ।

--without-kml \

--without-kriging $\backslash$

--with-cxxoptflags="-02 "

Once ISSM is configured correctly, the compilation can be triggered using the make install command. One can then test the ISSM JavaScript capabilities using the ISSM nightly run suite found in the test/NightlyRuns sub-directory of the ISSM trunk. All tests that end up with a JavaScript extension (.js) have been used for validation of the ISSM JavaScript capabilities. They provide a good starting point for users to build on. 